ARTIGO ORIGINAL ORIGINAL ARTICLE

Palavras-chave:

método de custeio baseado em atividade e tempo, Instituição de Longa Permanência para Idosos, custos de cuidados de saúde, custos diretos de serviços

\section{Aplicação do método de custeio baseado em atividade e tempo (TDABC) em uma Instituição de Longa Permanência de Idosos (ILPI)}

\author{
The application of time-driven activity-based \\ costing in an Elderly People Institution \\ Rafaela Caspary Roithmann', Karen Brasil Ruschel2, Ana Paula Beck da Silva Etges ${ }^{1,2}$ \\ DOI: 10.21115/JBES.v12.n1.p23-31
}

\begin{abstract}
RESUMO
Objetivos: $O$ envelhecimento da população brasileira é caracterizado por ser um processo progressivo e crescente. A necessidade de gerenciamento econômico robusto dos serviços disponibilizados à saúde do idoso torna-se imprescindível. O objetivo deste estudo foi avaliar a estrutura de gestão de custos de uma Instituição de Longa Permanência de Idosos (ILPI). Métodos: As orientações para a aplicação do método de custeio baseado em atividade e tempo (TDABC) foram utilizadas como método de análise de custos. Os dados dos anos de 2018 e 2019 foram considerados para formar as análises e viabilizar a compreensão dos diferentes graus de consumo de recursos, durante o ciclo de cuidados da ILPI. Resultados: A aplicação da metodologia estimou o custo unitário de cada residente considerando o seu grau de dependência devido a sua condição clínica. Pôde-se identificar pontos de melhorias gerenciais, com ênfase na revisão da alocação das capacidades de hospedagem. Conclusões: $\mathrm{OTDABC}$, como uma metodologia de fácil aplicação para estruturação dos custos relacionados a cada paciente, traz resultados oportunos para a melhor gestão e otimização de recursos em organizações de saúde. Sugere-se para trabalhos futuros a aplicação de um estudo de precificação dos serviços da ILPI, de modo que essa possa pautar os valores de mensalidades cobradas em bases mais sólidas.
\end{abstract}

\section{Keywords:}

time-driven activity-based costing, Elderly People Institution, healthcare costs, direct service costs

\begin{abstract}
Objectives: The need for a robust management of services focus on the elderly health becomes essential. The objective of this study was to evaluate the costs structure of an Elderly Long Term Care Institution (ILPI). Methods: The literature orientations for the application of the time-driven activity-based costing (TDABC) were used as a cost analysis method. Data from 2018 and 2019 were considered to form the analyzes and enable the understanding of the different degrees of resource consumption during the LTCI care cycle. Results: The application of the methodology estimated the unit cost of each resident considering their degree of dependence due to their clinical condition. Management improvement points could be identified, with emphasis on the review of hosting capacity allocation. Conclusions: TDABC as an easily applied methodology that brings timely results for better resource management and optimization in healthcare organizations. It is suggested for future researches the application of a pricing study of the services of ILPI, so that it can guide the monthly fees charged on a better substantiated basis.
\end{abstract}

Recebido em: 22/08/2019. Aprovado para publicação em: 24/03/2020.

1. Escola Politécnica da Pontifícia Universidade Católica do Rio Grande do Sul, Porto Alegre, RS, Brasil.

2. Instituto Nacional de Ciência e Tecnologia para Avaliação de Tecnologias em Saúde (INCT/IATS), Porto Alegre, RS, Brasil. Instituição em que o estudo foi realizado: Escola Politécnica da Pontifícia Universidade Católica do Rio Grande do Sul. Autor correspondente: Ana Paula Beck da Silva Etges. Instituto Nacional de Ciência e Tecnologia para Avaliação de Tecnologias em Saúde (INCT/IATS), Universidade Federal do Rio Grande do Sul (UFRGS). Ramiro Barcelos, 2.350, prédio 21-507, Porto Alegre, RS, Brasil. CEP: 90035-903. E-mail: aetges@hcpa.edu.br 


\section{Introdução}

O envelhecimento da população brasileira, caracterizado por ser um processo progressivo e crescente, é uma preocupação latente das áreas voltadas à saúde do idoso e às políticas sociais (Piuvezam et al., 2016). Resultado do conjunto das transições epidemiológica e demográfica - redução das altas taxas de natalidade e mortalidade (Giacomelli et al., 2016) -, esse cenário culminou no elevado aumento da proporção de idosos em relação à população total no Brasil (Nasri, 2008).

Essa proporcionalidade crescente na terceira idade foi evidenciada por meio do estudo das Projeções da População brasileira, realizado pelo Instituto Brasileiro de Geografia e Estatística (IBGE) em 2018. Estimou-se que 25,5\% (58,2 miIhões) da população brasileira terá 65 anos ou mais em 2060, enquanto no ano atual esse percentual se mantém em 9,2\% (19,2 milhões). Diante disso, surge a necessidade de alterações e adaptações no gerenciamento das estruturas e serviços disponibilizados à terceira idade, de modo que estejam cada vez mais consolidados e acessíveis e promovam a inclusão de todos os idosos (Piuvezam et al., 2016).

No intuito de atender às necessidades desse grupo etário, foram criadas as Instituições de Longa Permanência para Idosos (ILPI), que buscam promover as condições básicas de saúde e infraestrutura, visando, assim, à longevidade aliada à qualidade de vida dessa parcela da sociedade (Zacarias et al., 2008). A nomenclatura "ILPI" ficou comumente conhecida por meio da Sociedade Brasileira de Geriatria e Gerontologia (SBGG). A entidade, de caráter científico e sem fins lucrativos, adotou tal expressão com o objetivo de substituir o preconceito existente referente às casas geriátricas, anteriormente conhecidas como "asilos" (CNMP, 2016). Entretanto, constata-se que a escassez de gerenciamento dos recursos e a gama de custos associados desempenham influência direta na qualidade do serviço entregue aos idosos. Essa carência, aliada ao despreparo dos gestores, pode ser responsável por levar a instituição à decadência (Creutzberg et al., 2007).

Segundo a Resolução de Diretoria Colegiada (RDC) no 283/2005, as ILPIs são instituições de caráter residencial, destinadas em domicílio coletivo de pessoas com idade igual ou superior a 60 anos, em condições de liberdade, dignidade e cidadania. Podendo ser governamentais ou não governamentais, essas instituições possuem a responsabilidade de oferecer serviços nos âmbitos social, médico, psicológico, de enfermagem, odontologia, entre outras áreas da saúde que sejam de necessidade e dependência de seus residentes.

O Instituto de Pesquisas Econômicas Aplicadas (Ipea) realizou um levantamento das ILPIs no país e constatou a presença de 3.548 instituições no território brasileiro no ano de 2011. Contudo, devido à crescente população interessada e ao alto nível técnico requerido para a prática dos atendimentos, os custos associados a esse serviço são elevados (Scampini et al., 2018).
A elaboração de um sistema de custeio íntegro é essencial, visto que aquilo que não é medido de maneira adequada não pode ser controlado, gerenciado e, consequentemente, meIhorado (Kaplan \& Porter, 2011). Kaplan e Cooper (1998) identificam o modelo de custeio baseado em atividades (ABC) como sendo uma metodologia completa, projetada para informar a administração a respeito de seus exercícios econômicos passados, atuais e futuros. O objetivo desse sistema é a avaliação com precisão das atividades desenvolvidas em uma empresa. Por meio de direcionadores, o método visa alocar as despesas indiretas de forma mais realista aos serviços (Abbas et al., 2002).

Parte-se do princípio de que as atividades consomem recursos, enquanto produtos, clientes ou outros objetivos de custo consomem as atividades. Assim, essa metodologia traz uma proposta de valor significativo (Mauss \& Costi, 2004). Entretanto, o método ABC possui elevada complexidade de coleta de dados e metodologia de cálculo, bem como alto custo associado à sua implementação por completo, não sendo, portanto, comumente adotado pelas empresas (Jericó \& Castilho, 2010).

Como meio de atender às limitações da metodologia ABC, Kaplan e Anderson (2007) apresentam o método de custeio baseado em atividade e tempo (TDABC) como sendo uma ferramenta acessível e de aplicabilidade a diferentes setores, devido à sua implantação simplificada. A inovação do TDABC consistiu basicamente em possibilitar a elaboração das equações de tempo, visando à identificação das atividades mais demoradas e dos recursos mais custosos. Além disso, a metodologia viabiliza a utilização de um único direcionador - fatores ou variáveis empregadas nas equações - como forma de mensurar o custo de cada atividade: o tempo (Siguenza-Guzman et al., 2014).

No setor da saúde, a aplicabilidade do método TDABC vem sendo evidenciada em diversos estudos em instituições de tratamentos e cuidados médicos. Helmers e Kaplan (2016), em conjunto com a Universidade de Harvard e a Mayo Clinic, empregaram a metodologia de custeio aprimorada em seus procedimentos clínicos, obtendo êxito em demonstrar as oportunidades que o método traz ao basear os tratamentos médicos nos conceitos de valor e eficiência.

No âmbito das ILPIs, tal método traz simplicidade em sua aplicação para a medição e o gerenciamento dos custos, uma vez que permite a análise dos custos associados aos recursos relacionados a cada residente da instituição (McBain et al., 2016). Além disso, o TDABC traz benefícios para uma estruturação precisa, uma vez que atribui os custos dos recursos diretamente aos residentes e seus respectivos graus de dependência. Portanto, a condição médica de cada residente da instituição torna-se a unidade fundamental de análise para medir custos e resultados (Chou et al., 2018).

Este estudo tem como principal objetivo avaliar a estrutura de gestão de custos de uma ILPI, por meio do desenvolvimento e aplicação do método de custeio TDABC. 


\section{Métodos}

Estudo de caso que apresenta a aplicação do TDABC em uma ILPI privada, localizada na cidade de Porto Alegre, Rio Grande do Sul. Atualmente, a ILPI hospeda 64 idosos, possuindo 120 funcionários com vínculo empregatício salariado, separados por meio de suas especialidades, de forma a oferecer toda assistência médica e de apoio requerida aos residentes. O estudo de caso da instituição foi desenvolvido ao longo dos anos de 2018 e 2019, considerando os dados fornecidos pela própria ILPI como transparentes e com veracidade precisa.

Os dados foram submetidos à multiplicação por uma constante a fim de preservar o sigilo dos valores reais. Não foram considerados os custos dos serviços prestados de forma voluntária à instituição, incorporando somente aqueles com vínculo empregatício salariado na ILPI.

O método proposto utilizou como base os conceitos e definições descritos por Kaplan e Anderson (2007) na apresentação seminal do TDABC, e as etapas referentes à sua aplicação foram estruturadas por meio da adaptação dos passos descritos por da Silva Etges et al. (2019), de forma a guiar o estudo. Para tanto, o método de trabalho proposto foi dividido em cinco etapas principais, as quais foram desdobradas em subetapas de aplicação, conforme apresentado na Figura 1.

Etapa 1 - Contextualização: Iniciou-se com a definição do estudo na ILPI sob a perspectiva de uma instalação da saúde. Tal ótica é necessária para guiar o estudo com o foco no auxílio e melhora do gerenciamento dos recursos internos da organização. A clareza de entendimento da perspectiva a ser seguida orienta o processo de tomada de decisão para usuários internos da própria instituição.

A fim de facilitar o cuidado e o atendimento e seguindo as orientações da Agência Nacional de Vigilância Sanitária (Anvisa), os idosos são acomodados e separados nas instalações por meio da semelhança de seus graus de dependência, classificados pela Anvisa em: grau I, grau II e grau III.
Entendem-se como com dependência de grau I todos os idosos que são independentes em suas atividades diárias, mesmo que requeiram o uso de equipamentos de autoajuda. Os idosos que possuem dependência em até três atividades de autocuidado para a vida diária, mas que não possuem comprometimento cognitivo ou têm alteração cognitiva controlada são definidos como grau II. E, por fim, idosos com dependência que requeiram assistência em todas as atividades de autocuidado para a vida diária e/ou que possuam comprometimento cognitivo, são classificados como grau III (RDC n॰283, 2005).

Durante a primeira etapa, foram realizadas três reuniões com os responsáveis pelas áreas administrativa e financeira, com duração média de 2 a 4 horas cada uma, incluindo visitas guiadas pelas áreas da instituição. As reuniões foram pautadas por meio de conversas livres, de forma a compreender as características, estruturas e processos da ILPI e, ainda, definir o escopo exato do estudo. Já as visitas internas à instituição foram conduzidas por meio de funcionários do departamento de recursos humanos, sendo percorridas todas as instalações nas quais os idosos usufruem de suas atividades diárias.

A ILPI possui acomodações segregadas em três alas e cinco unidades de cuidados intensivos, denominadas, internamente, de: Ala Nova, Ala Apoio, Ala Leste, Unidade Semiprivativa IV, Unidade Semiprivativa V, Unidade Semiprivativa VI, Unidade Semiprivativa VII e unidade especializada em cuidados intensivos (Unitesp). Todas as instalações são equipadas para acomodar os residentes por completo.

No que diz respeito à separação dos residentes nas alas e unidades, a ILPI possui uma relação de autoria própria baseada nos três graus de dependência descritos. Define-se que idosos com grau l e grau II moderado residirão nas alas, enquanto os idosos com grau II avançado e/ou grau III moderado serão acomodados nas unidades. Por fim, residentes com grau III avançado serão alocados na Unitesp.

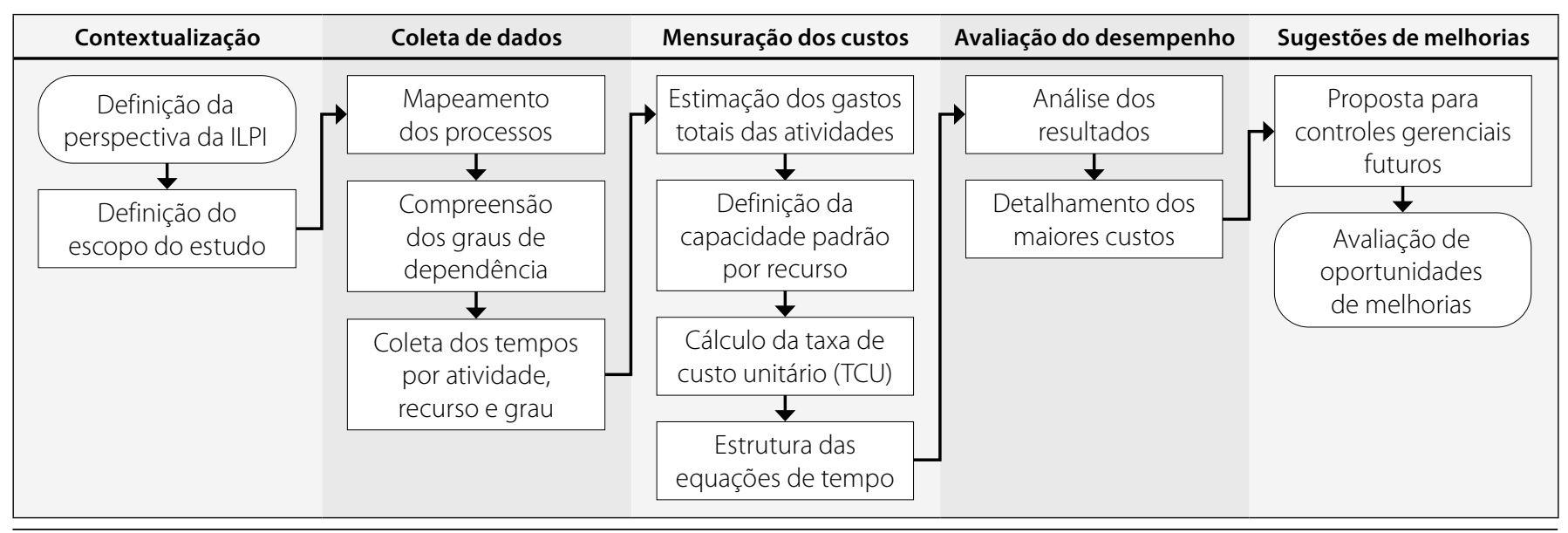

Figura 1. Fases do método de pesquisa. 
Etapa 2 - Coleta de Dados: Juntamente com a coordenadora financeira e por meio de conversas e observações, foi realizado o mapeamento do ciclo de atividades da instituição. Nesse contexto de ILPI, entende-se por mapear um ciclo a compreensão dos fluxos diários de cuidados e tratamentos aos residentes da instituição, utilizando como meio para tal: (i) identificação e descrição das atividades rotineiras; (ii) identificação dos grupos de recursos usados em cada uma das atividades - tanto as estruturas físicas necessárias quanto o pessoal envolvido para que as atividades sejam praticadas (Lacerda et al., 2017).

O ciclo de atividades e cuidados aos residentes foi desenhado partindo de macroatividades da instituição (Figura 2).

Hospedagem: atividades que o idoso exerce sem auxílio de profissionais da ILPI em sua rotina

Após a descrição e a compreensão do ciclo de atividades terem sido concluídas e validadas pela gerente administrativa da instituição, foram analisados os dados referentes aos custos totais mensais da ILPI. A instituição já possuía a estruturação de um Centro de Custos - desenvolvida nos anos anteriores com o apoio de uma empresa de contabilidade externa contratada. A Tabela 1 apresenta os custos totais mensais da ILPI como um todo - dados utilizados conforme fornecido pela instituição.

Ainda nessa segunda etapa da metodologia do trabalho, objetivou-se, também, compreender a divisão dos residentes por meio das oito categorias de acomodações associadas aos três graus de dependência definidos pela RDC nº 283, de 26 de

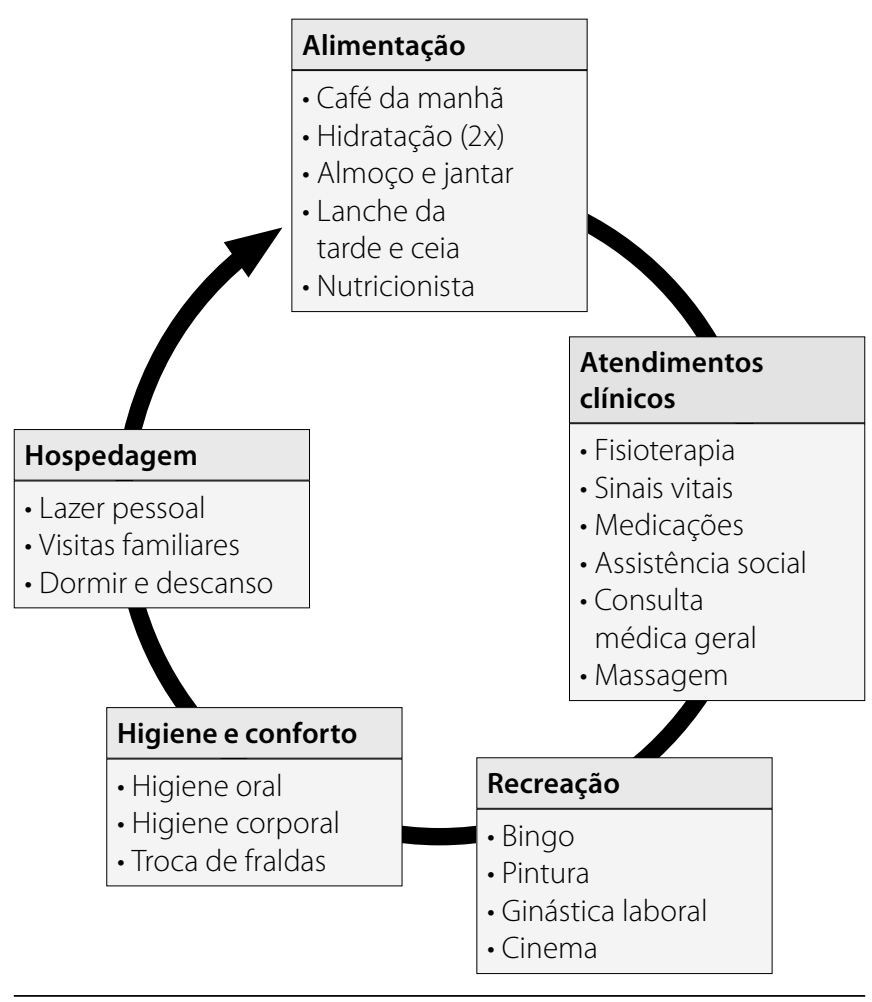

Figura 2. Ciclo de atividades e cuidados aos residentes.
Tabela 1. Custo total mensal da ILPI

\begin{tabular}{lc}
\hline Unidade de gasto & Total (R\$/mês) \\
\hline Coordenadoria administrativa & $84.803,03$ \\
\hline Coordenadoria de saúde & $2.400,78$ \\
\hline Coordenadoria operacional & $43.034,17$ \\
\hline Setor de alimentação & $58.596,65$ \\
\hline Aquisições gerais & $6.326,37$ \\
\hline Encargos de pessoal & $230.143,42$ \\
\hline Salário dos funcionários & $200.210,80$ \\
\hline Total & $\mathbf{6 2 5 . 5 1 5 , 2 2}$ \\
\hline
\end{tabular}

setembro de 2005, da Anvisa. A partir dessa classificação, realizou-se a coleta dos tempos consumidos por atividade, recursos e por grau de dependência dentro de cada acomodação. Para tanto, realizaram-se observações das atividades presentes no ciclo de cuidados e tratamentos de um idoso de cada grau preestabelecido, além de coleta de informações dos registros das enfermeiras responsáveis. Tais coletas foram segregadas por tipo de acomodação e por grau de dependência em um período de 30 dias.

Etapa 3 - Mensuração dos Custos: Inicia-se com a subetapa que consiste em estimar os gastos totais das atividades em valores monetários, quanto de cada tipo de recurso é utilizado individualmente pelos residentes, dentro de cada uma das oito categorias de acomodações disponíveis na ILPI.

Após, é realizada a definição das capacidades-padrão para cada recurso utilizado. Com os gastos totais das atividades previamente estimados e com as capacidades-padrão definidas, calcula-se a Taxa de Custo Unitário (TCU) de cada recurso. Assim, ao relacionar ambos os fatores mencionados acima, indica-se o custo por unidade de tempo de cada recurso envolvido no processo de cuidados aos residentes - fator que será utilizado posteriormente para a determinação do custo total por residente da ILPI (Keel et al., 2017).

A última subetapa dentro da Mensuração dos Custos teve como objetivo a estruturação das equações de tempo. Para tanto, foi determinado o tempo necessário no desenvolvimento de cada uma das atividades envolvidas no fluxo de tratamentos e cuidados ao idoso.

Ressalta-se que as coletas de tempo foram realizadas por cronoanálise do consumo de cada um dos 64 residentes na ILPI, associado ao seu grau de dependência e respectivo tipo de acomodação.

Etapa 4 - Avaliação do Desempenho: Por meio da exploração de gráficos e tabelas visuais, o estudo confrontou as informações de custos fornecidas pela ILPI no início do processo, com os resultados por meio da aplicação da metodologia TDABC. Junto com isso, com a participação dos responsáveis pelas áreas administrativa e financeira, foi realizado o detalha- 
mento dos maiores custos associados ao nível de cada residente por seu respectivo grau de dependência.

Etapa 5 - Sugestões de Melhorias: Em conjunto com os responsáveis da instituição, foram geradas as propostas para controles gerenciais futuros. A avaliação de oportunidades de melhorias nos controles gerenciais e a geração de dados mais exatos para a tomada de decisão foram a pauta na reunião de encerramento do estudo na ILPI.

\section{Resultados}

Os resultados foram conduzidos a partir do ciclo de atividades e levantamento inicial dos dados financeiros existentes na ILPI. Para que os custos controlados mensalmente fossem atribuídos aos pacientes, a primeira etapa se concentrou na análise dos tempos consumidos pelos pacientes em cada etapa do ciclo. Os resultados da coleta de tempos, em horas de consumo, das principais subatividades dentro de cada macroatividade definida estão compilados na Tabela 2.

\section{Mensuração de custos}

Entende-se que o custo total mensal da ILPI de R\$ 625.515,22 detalhado na Tabela 1 - é oriundo da prática e recorrência das subatividades que compõem as cinco macroatividades consumidas diariamente pelos recursos e residentes da instituição. Diante desse cenário, como meio de ratear os custos totais mensais da instituição por meio das cinco macroatividades do ciclo definido, utilizou-se a representatividade de consumo dos residentes - em horas de utilização - em cada macroatividade da instituição separadamente. Para tanto, baseou-se no somatório registrado na Tabela 2, sobre o valor total de consumo. A Tabela 3 destaca a representatividade de cada macroatividade, sendo a base para a Tabela 4, a qual demonstra tal relação de consumo, em horas, com os custos totais mensais atribuídos à cada macroatividade.

Após a compreensão do custo total mensal de cada uma das cinco macroatividades da ILPI, foi realizada a análise das capacidades-padrão para cada recurso existente e, na sequência,
Tabela 3. Representatividade de consumo das macroatividades

\begin{tabular}{lcc}
\hline Macroatividades & $\begin{array}{c}\text { Consumo total } \\
\text { (horas/mês) }\end{array}$ & $\begin{array}{c}\text { Representatividade } \\
\text { de consumo }\end{array}$ \\
\hline Higienização & 3.015 & $6,54 \%$ \\
\hline Alimentação & 5.450 & $11,83 \%$ \\
\hline Recreação & 1.900 & $4,12 \%$ \\
\hline Atendimentos médicos & 4.860 & $10,55 \%$ \\
\hline Hospedagem & 30.855 & $\mathbf{6 6 , 9 6 \%}$ \\
\hline Total & $\mathbf{4 6 . 0 8 0}$ & $\mathbf{1 0 0 \%}$ \\
\hline
\end{tabular}

calculada a taxa de custo de capacidade associada a cada macroatividade. $O$ estudo adotou como base um mês de 30 dias, considerando as 24 horas completas de um dia. A Tabela 5 demonstra a capacidade mensal resultante por macroatividade.

Seguido da realização da alocação do custo total mensal para cada macroatividade da ILPI, em conjunto com a conclusão do cálculo das capacidades-padrão, calcula-se a Taxa de Custo Unitário (TCU) de cada macroatividade, obtendo o quociente entre a divisão dos custos totais alocados pelas capacidades calculadas. A Tabela 6 indica o custo por unidade de tempo de cada macroatividade envolvida no ciclo de atividades e cuidados aos residentes.

Finalizando a etapa de Mensuração dos Custos, realiza-se a estruturação das equações de tempo. Por meio delas, o presente estudo estimou os custos individuais por paciente e associou-os aos graus de dependência e aos tipos de acomodações da qual o idoso faz parte. A Tabela 7 apresenta o custo por macroatividade alocado em cada uma das três alas e cinco unidades, subdividido entre o número de residentes atrelados aos três graus de dependência.

\section{Avaliação do desempenho}

A aplicação da metodologia TDABC viabilizou a estimativa dos custos em nível de cada residente com seu respectivo grau de dependência. Por meio da compreensão dos custos totais das macroatividades, foi possível converter as horas de consumo

Tabela 2. Tempo médio de consumo das macroatividades

\begin{tabular}{|c|c|c|c|c|c|c|}
\hline \multirow[b]{2}{*}{ Acomodações } & \multicolumn{6}{|c|}{ Tempo de consumo por macroárea (horas/mês) } \\
\hline & Higienização & Alimentação & Recreação & $\begin{array}{l}\text { Atendimentos } \\
\text { médicos }\end{array}$ & Hospedagem & Total \\
\hline Ala nova & 186 & 469,5 & 456,7 & 405 & $4.242,8$ & 5.760 \\
\hline Ala apoio & 120 & 276,5 & 118,7 & 202 & $2.162,8$ & 2.880 \\
\hline Ala leste & 119 & 226,5 & 134 & 199 & $2.201,5$ & 2.880 \\
\hline Unidade semiprivativa IV & 282,5 & 451,5 & 291,3 & 377 & $3.637,7$ & 5.040 \\
\hline Unidade semiprivativa $\mathrm{VI}$ & 279 & 455 & 192 & 439 & 2.955 & 4.320 \\
\hline Unidade semiprivativa VII & 525 & 697 & 212,7 & 835 & $4.930,3$ & 7.200 \\
\hline Unitesp & 1.180 & 2.164 & 220 & 1.759 & $6.917,0$ & 12.240 \\
\hline Total (horas) & 3.015 & 5.450 & 1.900 & 4.860 & 30.855 & 46.080 \\
\hline
\end{tabular}


individuais dos residentes em custos unitários. Ainda, o estudo viabilizou a identificação das atividades que consomem a maior parte dos gastos mensais da ILPI.

Obteve-se como resultado inicial a compreensão quantitativa dos distintos modos de consumo no decorrer do ciclo de atividades e cuidados praticados diariamente na institui- ção. Diante desse cenário, os valores calculados durante a subetapa de Mensuração dos Custos foram convertidos em informação e conhecimento a partir da realização de duas análises de resultados principais, sendo esses referentes aos custos totais por macroatividade e aos custos totais por grau de dependência.

Tabela 4. Custo total mensal por macroatividade (R\$)

\begin{tabular}{|c|c|c|c|c|c|}
\hline Unidade de gasto & Higienização & Alimentação & Recreação & $\begin{array}{l}\text { Atendimentos } \\
\text { médicos }\end{array}$ & Hospedagem \\
\hline Coordenadoria administrativa & $5.548,64$ & $10.029,87$ & $3.496,65$ & $8.944,07$ & $56.783,80$ \\
\hline Coordenadoria operacional & $3.585,05$ & $6.480,45$ & $2.259,24$ & $5.778,89$ & $36.688,83$ \\
\hline Setor de alimentação & - & $46.838,36$ & - & - & - \\
\hline Encargos de pessoal & $15.058,21$ & $27.219,65$ & $9.489,42$ & $24.272,94$ & $154.103,19$ \\
\hline Salário dos funcionários & $13.099,73$ & $23.679,45$ & $8.255,22$ & $21.115,98$ & $135.060,42$ \\
\hline Total & $37.705,56$ & $114.996,01$ & $26.039,66$ & $60.901,62$ & $385.876,36$ \\
\hline
\end{tabular}

Tabela 5. Capacidade mensal em horas dos recursos

\begin{tabular}{lc}
\hline Macroárea & Capacidade total (horas/mês) \\
\hline Higienização & 3.015 \\
\hline Alimentação & 5.450 \\
\hline Recreação & 1.900 \\
\hline Atendimentos médicos & 4.860 \\
\hline Hospedagem & 30.855 \\
\hline Total & $\mathbf{4 6 . 0 8 0}$ \\
\hline
\end{tabular}

Tabela 6. Taxas de Custo Unitário (TCUs) das macroatividades

\begin{tabular}{lc}
\hline Macroárea & TCU (R\$⿳/hora) \\
\hline Higienização & 12,51 \\
\hline Alimentação & 21,10 \\
\hline Recreação & 13,71 \\
\hline Atendimentos médicos & 12,53 \\
\hline Hospedagem & 12,51 \\
\hline Total & $\mathbf{7 2 , 3 6}$ \\
\hline
\end{tabular}

Tabela 7. Custo mensal por residente, grau de dependência e acomodação nas macroatividades (R\$)

\begin{tabular}{|c|c|c|c|c|c|c|c|}
\hline Acomodações & & Higienização & Alimentação & Recreação & $\begin{array}{l}\text { Atendimentos } \\
\text { médicos }\end{array}$ & Hospedagem & Total \\
\hline \multirow[t]{2}{*}{ Ala nova } & Gl & 200,10 & $1.101,43$ & 456,84 & 621,55 & $7.114,24$ & $9.494,15$ \\
\hline & Gll & 441,88 & $1.466,46$ & $1.324,82$ & 655,80 & $5.829,88$ & $9.718,84$ \\
\hline \multirow[t]{2}{*}{ Ala apoio } & Gl & 281,38 & $1.339,86$ & 405,06 & 639,09 & $6.921,37$ & $9.586,77$ \\
\hline & Gll & 656,56 & $1.814,62$ & 411,15 & 614,03 & $6.284,26$ & $9.780,62$ \\
\hline Ala leste & Gl & 273,05 & $1.086,66$ & 459,88 & 639,09 & $7.029,76$ & $9.488,44$ \\
\hline \multirow[t]{2}{*}{ Unidade semiprivativa IV } & Gll & 492,94 & $1.285,35$ & 561,91 & 622,38 & $6.615,67$ & $9.578,26$ \\
\hline & GIII & 575,28 & $1.814,62$ & 621,30 & 989,96 & $5.798,61$ & $9.799,76$ \\
\hline \multirow[t]{2}{*}{ Unidade semiprivativa $V$} & Gll & 456,47 & $1.455,91$ & 635,00 & 883,45 & $6.223,82$ & $9.654,65$ \\
\hline & GIII & 522,13 & $2.011,55$ & 415,72 & $1.050,53$ & $5.862,18$ & $9.862,11$ \\
\hline Unidade semiprivativa VII & GIII & 753,49 & $1.714,39$ & 340,34 & $1.062,02$ & $5.864,27$ & $9.734,51$ \\
\hline \multirow[t]{2}{*}{ Unitesp } & Gll & 619,05 & $1.577,24$ & 246,69 & $1.315,78$ & $5.912,21$ & $9.670,96$ \\
\hline & GIII & 901,27 & $2.833,75$ & 168,12 & $1.294,05$ & $4.978,64$ & $10.175,83$ \\
\hline Total (horas) & & $8.672,70$ & $25.869,88$ & $7.456,94$ & $13.952,44$ & $99.334,46$ & $155.286,42$ \\
\hline
\end{tabular}


A primeira análise representa o custo total utilizado para a realização das atividades que compõem o ciclo de cuidados aos residentes. Tal resultado auxilia na transparência para alocação dos valores da instituição, uma vez que, identificada a atividade que consome mais recursos, a ILPI pode planejar-se internamente no que tange a investimentos de valores, não superestimando investimentos em atividades de consumo secundário. Para tanto, os dados foram plotados em um Diagrama de Pareto, conforme a Figura 3, de modo que permitissem a melhor visualização da concentração dos custos de maior representatividade.

Conforme demonstrado na Figura 3, a hospedagem é a macroatividade que possui maior custo associado para a ILPI, devido ao grande número de horas consumidas em suas subatividades. Essa é a macroatividade que apresenta menor valor de TCU associado (R\$ 12,51/h), sendo até mesmo inferior à TCU da Recreação (R\$ 13,71) - macroatividade que representa menor custos à ILPI entre as cinco macroatividades comparadas.

A segunda análise ilustra a representação do custo total unitário para os três graus de dependência segregados nas acomodações específicas de cada categorização. Compreender que cada residente consome os recursos disponíveis em proporções distintas é ponto-chave para uma estruturação e gerenciamento robusto de custos de uma instalação da saúde. Atualmente a instituição estudada realiza somente uma precificação dos residentes por meio de mensalidades fixas por tipo de acomodação e, por isso, não consegue atentar-se a quanto esses idosos realmente custam, o que causa inconsistências nas conciliações de caixa no final do mês da ILPI.

A aplicação do TDABC demonstrou quais são os idosos que representam maiores custos à instituição, bem como onde eles estão acomodados entre as alas e unidades disponíveis. A Figura 4 demonstra a relação criada entre o custo de grau de dependência e a respectiva acomodação. Em complemento, a Tabela 8 detalha a representatividade de horas de consumo de cada acomodação. Assim, identifica-se quem são os residentes mais custosos para a ILPI, sendo, consequentemente, aqueles que requerem e demandam mais tempo de cuidados mensais.

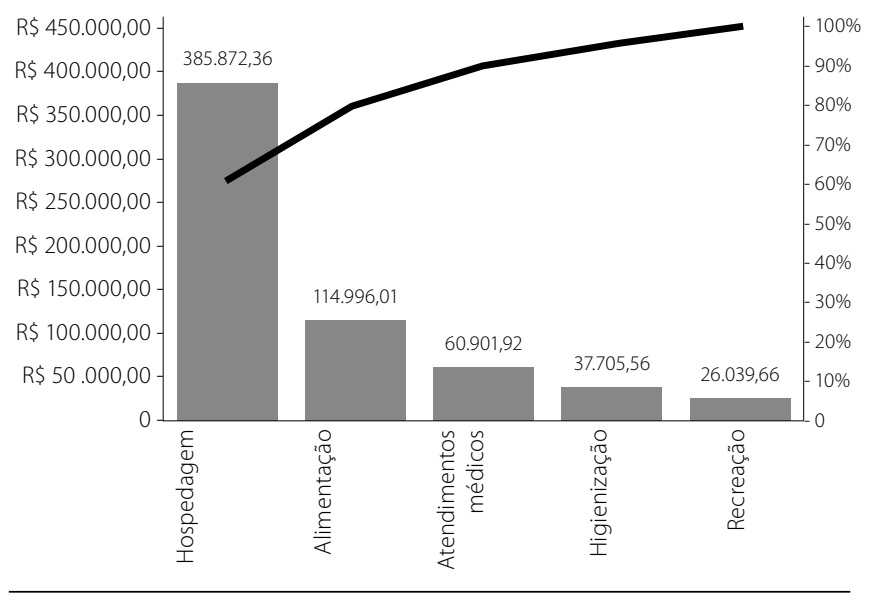

Figura 3. Representatividade de custo por macroatividade.

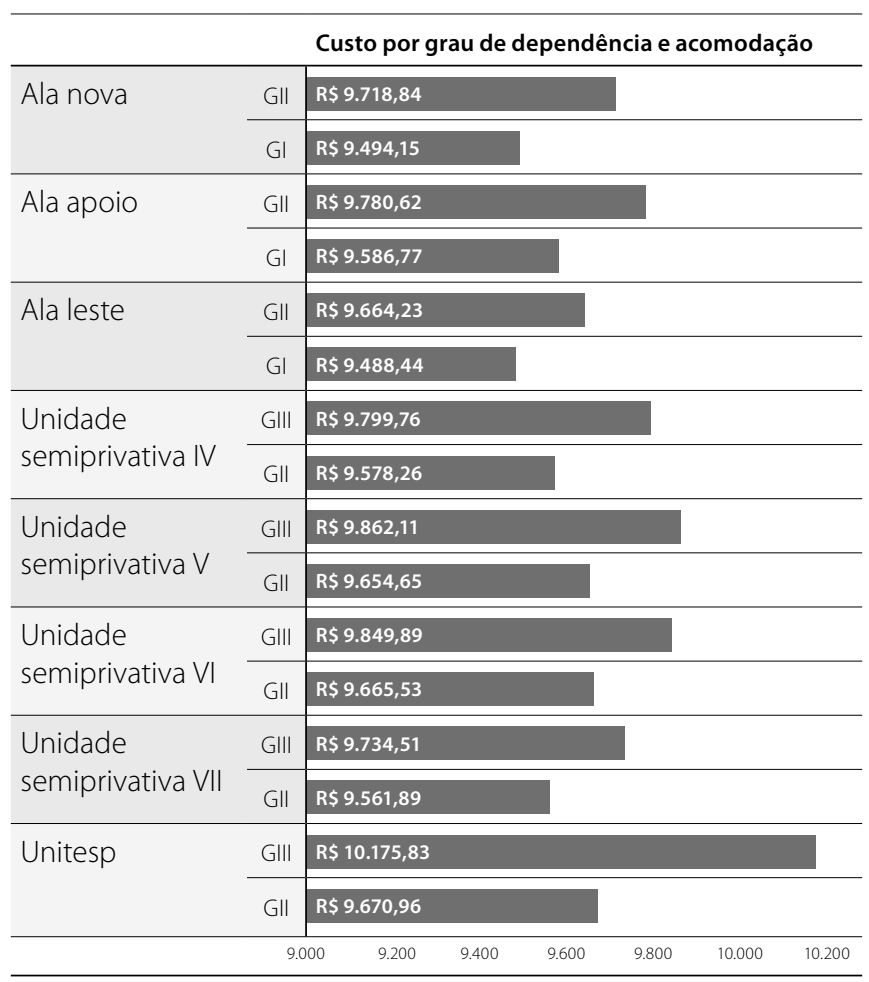

Figura 4. Representação de custo mensal por grau de dependência e acomodação.

Tabela 8. Representatividade de horas de consumo das acomodações

\begin{tabular}{lccc}
\hline Acomodações & Número de residentes & $\begin{array}{c}\text { Hora de consumo } \\
\text { total (horas/mês) }\end{array}$ & Representatividade \\
\hline Ala nova & 8 & 5.760 & $13 \%$ \\
\hline Ala apoio & 4 & 2.880 & $6 \%$ \\
\hline Ala leste & 4 & 2.880 & $6 \%$ \\
\hline Unidade semiprivativa IV & 7 & 5.040 & $11 \%$ \\
\hline Unidade semiprivativa V & 8 & 5.760 & $13 \%$ \\
\hline Unidade semiprivativa VI & 6 & 4.320 & $9 \%$ \\
\hline Unidade semiprivativa VII & 10 & 7.200 & $16 \%$ \\
\hline Unitesp & 17 & 12.240 & $\mathbf{2 7 \%}$ \\
\hline Total & $\mathbf{6 4}$ & $\mathbf{4 6 . 0 8 0}$ & $\mathbf{1 0 0 \%}$ \\
\hline
\end{tabular}


Por meio da interpretação dos custos discriminados acima, constata-se a vantagem da aplicação do método TDABC, uma vez que ele permite a fácil comparação dos custos dos diferentes residentes da ILPI. Esse viabiliza também a identificação de padrões de gastos por meio dos graus de dependência semelhantes. Ainda, torna-se transparente a identificação dos idosos mais custosos para a instituição e em que tipo de acomodação (alas e unidades) eles estão alocados.

Interpretando o gráfico, é possível compreender que os residentes de grau de dependência III, acomodados na Unitesp, são os idosos que representam o maior custo unitário mensal para a instituição, representando um valor de $\mathrm{R} \$ 10.175,83$ por mês. Tal fato é evidenciado pela análise dos tempos de consumo desses residentes, que representam, juntos, 27\% das 46.080 horas totais disponíveis por mês.

Avalia-se, também, que os idosos de grau I, de maneira geral, são menos custosos para a ILPI, constatando que os residentes com custos inferiores para a ILPI estão acomodados na Ala Leste, a qual representa $6 \%$ de consumo das horas totais disponíveis. Por fim, compreende-se que os idosos de grau ॥ podem representar custos semelhantes aos de grau I quando acomodados nas alas. Atualmente, os recursos são disponibilizados com base no número de residentes por acomodação, o que resulta em capacidades de recursos semelhantes nas alas. Tal fator influencia para que não se constatem grandes diferenças nos custos por grau de dependência I e II, uma vez que os idosos que residem nas alas possuem pequenas distinções físicas entre si, sem a incidência de perdas ou danos cognitivos.

Por fim, o modelo aplicado comprovou que os custos individuais estão diretamente relacionados com o grau de dependência dos residentes, bem como com a capacidade de recursos fornecida para cada tipo de acomodação. Assim, avalia-se a aplicação do TDABC com desempenho positivo, uma vez que foram possíveis tanto a identificação dos custos por cada macroatividade quanto a visualização concreta das proporções de consumo dos residentes.

\section{Sugestão de melhorias}

Estimar o custo relacionado a cada residente é de extrema importância para a tomada de decisão das áreas administrativas da instituição, sendo esse um caminho essencial para melhorar o desempenho econômico da organização. A carência de uma estrutura de custeio eficaz, traduzida pelo modo raso em que são levantados os custos da ILPI atualmente, culminam na falta de transparência da realidade da instituição, bem como em inconsistências no fechamento mensal.

Uma sugestão de melhoria foi identificada partindo da análise realizada durante a subetapa de Avalição de Desempenho. Esta demonstrou que não há diferenças significativas de custos para os residentes de diferentes graus de dependência acomodados nas alas na instituição, uma vez que eles possuem capacidades instaladas semelhantes. Ainda, nota-se que, ao somar a representatividade, em horas consumidas das macroatividades, das Alas Leste e de Apoio, identifica-se que elas consomem, juntas, o mesmo consumido pela Ala Nova sozinha, sendo que as três alas possuem os mesmos recursos disponibilizados.

Avaliando a disposição dos quartos contidos nas alas, nota-se que há quartos vagos em suas dependências, o que representa perda por ociosidade de capacidade de hospedagem para a instituição. A Tabela 9 demonstra a taxa de ocupação e a consequente perda pela não utilização da capacidade completa de hospedagem das alas.

Tabela 9. Taxa de ocupação das alas

\begin{tabular}{lccc}
\hline Acomodações & $\begin{array}{c}\text { Quartos } \\
\text { disponíveis }\end{array}$ & $\begin{array}{c}\text { Quartos } \\
\text { utilizados }\end{array}$ & $\begin{array}{c}\text { Taxa de } \\
\text { ocupação }\end{array}$ \\
\hline Ala nova & 15 & 8 & $53 \%$ \\
\hline Ala apoio & 8 & 4 & $50 \%$ \\
\hline Ala leste & 6 & 4 & $67 \%$ \\
\hline Total & $\mathbf{2 9}$ & $\mathbf{1 6}$ & $\mathbf{5 5 \%}$ \\
\hline
\end{tabular}

\section{Conclusões}

A gestão da ILPI estudada identificou a necessidade latente de uma estruturação de custos pautada em métodos que forneçam a transparência e a exatidão dos seus resultados financeiros. A partir da análise de custos, a ILPI poderá realizar as priorizações de investimentos, bem como aprimorar a assertividade de ações que visem à otimização de custos e recursos.

Nesse contexto, justifica-se a aplicação do método TDABC como meio de transformar dados em informações que gerem conhecimento e, portanto, auxiliem a área administrativa e gerencial a tomar de decisões.

O desenvolvimento e a aplicação do TDABC viabilizaram a avaliação da estrutura de gerenciamento dos custos da ILPI por proporcionar a compreensão dos custos unitários por grau de dependência. Mapear o ciclo de atividades e cuidados aos residentes, bem como realizar a coleta dos tempos de consumo, possibilitou um melhor entendimento do modo de alocação de recursos.

Sugere-se para trabalhos futuros a aplicação de um estudo de precificação dos serviços praticados pela ILPI, de forma que a instituição possa pautar os valores de mensalidades cobradas em bases mais sólidas.

\section{Referências bibliográficas}

Abbas K, Lezana AGR, Menezes EA. Apuração dos custos nas organizações hospitalares: o método $A B C$ aplicado no serviço de processamento de roupas de um hospital. Rev FAE. 2002;5(2). 
Ching HY. Gestão baseada em custeio por atividades: ABM - Activity Based Management. São Paulo: Atlas; 1997.

Chou J, Shah M, Watts A, Gardiner M, Kaplan R, Miller J, et al. TDABC Cost Analysis of Ocular Disorders in an Ophthalmology Emergency Department versus Urgent Care: Clinical Experience at Massachusetts Eye and Ear. J Acad Ophthalmol. 2018;10(01):E55-60.

Creutzberg M, Gonçalves LHT, Sobottka EA, Ojeda BS. A instituição de longa permanência para idosos e o sistema de saúde. Rev Latino-Am Enfermagem. 2007;15(6):1144-9.

Conselho Nacional do Ministério Público (CNMP). Manual de atuação funcional: o Ministério Público na Fiscalização das Instituições de Longa Permanência para Idosos. Brasília: CNMP; 2016.

da Silva Etges APB, Cruz LN, Notti RK, Neyeloff JL, Schlatter RP, Astigarraga CC, et al. An 8-step framework for implementing time-driven activity-based costing in healthcare studies. Eur J Health Econ. 2019;20(8):1133-45.

Giacomelli GS, Chiapinoto FV, Marion Filho PJ, Vieira KM. Transição demográfica e gasto público: uma análise comparativa de diferentes contextos. Rev Estud Soc. 2016;18(37):164-81.

Helmers RA, Kaplan RS. TDABC in primary care: results of a Harvard/Mayo Clinic collaboration. Healthc Financ Manage. 2016;70(7):35-41.

Instituto Brasileiro de Geografia e Estatística (IBGE). Projeção da População 2018. Available from: https://www.ibge.gov.br/apps/populacao/projecao/. Accessed on: Aug 28, 2018.

Instituto de Pesquisa Econômica Aplicada (Ipea). Infraestrutura Social e Urbana no Brasil: subsídios para uma agenda de pesquisa e formulação de políticas públicas, 2011. Available from: http://www.ipea.gov.br/ portal/images/stories/PDFs/comunicado/110524_comunicadoipea93.pdf. Accessed on: Set 7, 2018.

Jericó MC, Castilho V. Gerenciamento de custos: Aplicação do método de custeio baseado em atividades em centro de material esterilizado. Rev Esc Enferm USP. 2010;44(3):745-52.

Kaplan RS, Anderson SR. Time-driven activity-based costing: a simpler and more powerful path to higher profits. Boston: Harvard Business School Publishing Corporation; 2007. v. 82.

Kaplan RS, Porter ME. How to solve the cost crisis in health care. Harv Bus Rev. 2011;89(9):46-52, 54, 56-61 passim.
Kaplan RS, Cooper R. Cost and Effect: Using Integrated Cost Systems to Drive Profitability and Performance. Boston: Harvard Business School Press; 1998.

Keel G, Savage C, Rafiq M, Mazzocato P. Time-driven activity-based costing in health care: A systematic review of the literature. Health Policy. 2017;121(7):755-63.

Lacerda TTB, Horta NC, Souza MCMR, Oliveira TRPR, Marcelino KGS, Ferreira QN. Characterization of long-term care facilities for the elderly in the metropolitan region of Belo Horizonte. Rev Bras Geriatr Gerontol. 2017;20(6):743-53.

Mauss CV, Costi RM. O Método de Custeio ABC como instrumento de gestão. São Leopoldo: Atlas; 2004.

McBain RK, Jerome G, Warsh J, Browning M, Mistry B, Faure PAl, et al. Rethinking the cost of healthcare in low-resource settings: the value of time-driven activity-based costing. BMJ Glob Health. 2016;1(3).

Nasri F. O envelhecimento populacional no Brasil. Einstein. 2008;6(Supl 1):S4-6.

Piuvezam G, Lima KC, Carvalho MS, Xavier VGP, Silva RA, Dantas ARF, et al. Atenção primária à saúde e os idosos institucionalizados: a perspectiva da gestão municipal no Brasil. Rev Port Saúde Pública. 2016;34(1):92-100.

Resolução de Diretoria Colegiada - RDC n²83, de 26 de setembro de 2005. Instituição de Longa Permanência de Idosos. Agência Nacional de Vigilância Sanitária - Anvisa. Available from: http://portal.anvisa.gov.br/ documents/10181/2718376/RDC_283_2005_COMP.pdf/a38f2055-c23a4eca-94ed-76fa43acbldf. Accessed on: Fev 20, 2019.

Scampini GF, Silva IABP, Anjos VML, Vieira RTV. Contabilidade Gerencial e de Custos: um estudo de caso da Associação Asilo São João Bosco AASJB. REPAE. 2018;4(1):48-69.

Siguenza-Guzman L, Van den Abbeele A, Leuven K. Time-Driven ActivityBased Costing systems for cataloguing processes: a case study. Liber Quart. 2014;23(2):160-86.

Zacarias FLB, Leonardo VS, Silva AT, Borinelli ML. Proposta de apuração e evidenciação de custos em entidades do terceiro setor: aplicação do custeio integral em uma instituição de longa permanência de idosos. In: Anais do Congresso Brasileiro de Custos-ABC; 2008. 